\title{
The effect of single periocular injection of methylprednisolone and drainage of suprachoroidal fluid in the treatment of rhegmatogenous retinal detachment combined with choroidal detachment
}

\author{
Yong $\mathrm{Wei}^{1,2} \cdot$ RongLe $\mathrm{Zhou}^{3} \cdot \mathrm{Xiaobo}^{\mathrm{Wang}}{ }^{2} \cdot$ Xiuju Chen ${ }^{2} \cdot$ Hao Chen ${ }^{1}$
}

Received: 19 June 2018 / Revised: 16 February 2019 / Accepted: 22 February 2019 / Published online: 2 April 2019

(c) The Royal College of Ophthalmologists 2019

\begin{abstract}
Purpose In this study we compared the anatomic and functional outcomes of two steroid treatments on rhegmatogenous retinal detachment (RRD) combined with choroidal detachment $(\mathrm{CD})$, namely treatment with oral prednisolone $(1 \mathrm{mg} / \mathrm{kg}$ daily) for 3-7 days before vitrectomy or a single periocular injection of methylprednisolone (40 mg) 1-3 days before vitrectomy. We also analyzed the outcomes of the eyes with subsided $\mathrm{CD}$ and the eyes with persistent $\mathrm{CD}$ that underwent drainage of suprachoroidal fluids during the vitrectomy.

Methods This was a prospective randomized study. Seventy five eyes with RRD combined with CD were divided into 2 groups based on the two different treatment regimens as above. The eyes in each group were further divided into 2 subgroups (A: CD subsided eyes; $\mathrm{B}$ : CD persistent eyes) according to the response of CD to the treatment of steroids. Retinal reattachment rates were measured at 6 months after the removal of silicone oil.

Results At 6 months after silicone oil removal, the retinal reattachment rate was similar $(p=0.666)$ in the oral prednisolone group $(91.7 \%, 33 / 36)$ and the periocular injection group $(94.9 \%, 37 / 39)$. Similar retinal reattachment rates $(p=0.364)$ were also found in the CD subsided eyes $(97.1 \%, 34 / 35)$ and the CD persistent eyes $(90.0 \%, 36 / 40)$. The retinal reattachment rate was comparable among the subgroups $(p=0.395$; oral prednisolone A group: $95.2 \%, 20 / 21$; oral prednisolone B group: 86.7\%, 13/15; periocular injection A group: 100\%, 14/14; periocular injection B group: 92.0\%, 23/25).

Conclusions For RRD combined with CD, eyes treated with a single periocular injection of methylprednisolone ( $40 \mathrm{mg}$, 1-3 days before pars plana vitrectomy) combined with the drainage of suprachoroidal fluids during the surgery had similar anatomic and functional outcomes compared to the eyes treated with oral prednisolone for 3-7 days before vitrectomy.
\end{abstract}

\section{Introduction}

The occurrence of choroidal detachment (CD) in the eyes with primary rhegmatogenous retinal detachment (RRD) is

Yong Wei

weiyongdoctor@163.com

1 The Eye Hospital of Wenzhou Medical University, Wenzhou, Zhejiang 325027, China

2 Xiamen Eye Center Affiliated to Xiamen University, Xiamen 361000, China

3 Shaanxi Ophthalmic Medical Center, Xi'an No.4 Hospital, Affiliated Guangren Hospital, School of Medicine, Xi'an Jiaotong University, Xi' an 710004, China rare, ranging from 2 to $4.5 \%$ in western countries and 4.2 to $18.1 \%$ in China [1-3], respectively. For RRD combined with $\mathrm{CD}$, the retinal reattachment rate after conventional scleral buckling remains low, varying from 35.4 to $52.4 \%$ [3-5], due to the high incidence of proliferative vitreoretinopathy (PVR) after surgery. The treatment of preoperative oral steroids combined with pars plana vitrectomy (PPV) has shown to improve the overall retinal reattachment rate in the eyes with RRD combined with CD [6-9].

In our previous study, we administered systemic (oral prednisolone) or local (periocular/intravitreal) corticosteroids for 3-7 days in patients with RRD combined with CD before PPV. We found that the periocular/intravitreal administration of corticosteroids was an acceptable alternative for the patients who could not tolerate the systemic steroids [10]. If persistent CD did not subside completely 
after 7 days of systemic steroid treatment, we performed the suprachoroidal fluid drainage through a pars plana sclerotomy during vitrectomy. We found that the retinal reattachment rate in the eyes with persistent CD seemed to be similar to that of the eyes with CD subsided completely within 7 days.

In another group of patients with RRD combined with $\mathrm{CD}$, we administered a single periocular injection of methylprednisolone $(40 \mathrm{mg}$ ) for only $1-3$ days before PPV. For the eyes with persistent $\mathrm{CD}$ that did not subside completely within 3 days, we performed the suprachoroidal fluid drainage through a pars plana sclerotomy during vitrectomy. We hypothesized that if the single preoperative periocular injection of methylprednisolone resulted in similar retinal reattachment rates compared to the 7-day preoperative systemic steroid treatment, it would not be necessary to delay the surgery and use systemic steroids before PPV.

In this study we compared the anatomic and functional outcomes of two steroid treatments for the patients with RRD combined with $\mathrm{CD}$, the treatment of oral prednisolone ( $1 \mathrm{mg} / \mathrm{kg}$ daily) for 3-7 days before vitrectomy or a single periocular injection of methylprednisolone $(40 \mathrm{mg}) 1-$ 3 days before PPV. We also compared the outcomes of the eyes with $C D$ that subsided before vitrectomy and the eyes with persistent $C D$ that did not subside before surgery and underwent the drainage of suprachoroidal fluids during the surgery.

\section{Patients and methods}

This was a prospective randomized study. This study was approved by the appropriate institutional review board and was performed in accordance of the Declaration of Helsinki of the World Medical Association. A total of 75 consecutive patients (75 eyes) underwent a PPV from November 2011 to May 2016 were recruited in this study. The timing of the vitrectomy was depended on the subsiding of the CD. All surgeries were performed within a week following the treatment of steroids. All patients presented RRD, CD, hypotony and vitritis. Preoperative $\mathrm{CD}$ was diagnosed by either indirect ophthalmoscopic examination or B-scan ultrasonography. The severity of $\mathrm{CD}$ was classified as mild (if the shallow peripheral choroidals were present), severe (if the detachment reaches up to the equator), and moderate (if the detachment was in the intermediate extent) [6], respectively. The information collected from each patient included age, gender, initial visual acuity, clinical manifestations, surgical procedures, postoperative complications, and final visual outcome. Patients were excluded from the study if they had any of the following conditions: recurrent retinal detachment after primary scleral buckling, history of previous vitreoretinal surgery, history of openglobe ocular trauma, requiring primary vitrectomy for macular hole, giant retinal tear, nonclearing vitreous hemorrhage or grade $\mathrm{C}$ proliferative vitreoretinopathy. The potential merits and risks of treatment procedures were explained to the patients before the surgery. The informed consent was obtained from all patients.

The vitrectomy technique entailed a 20-gauge/23-gauge/ 25-gauge 3-port pars plana approach for a sclerotomy located 3 to $3.5 \mathrm{~mm}$ posterior to the limbus. As described preciously [10], phacoemulsification was performed before PPV if the view of the fundus was obscured by the lens opacities. For the 20-gauge 3-port pars plana approach, a long infusion cannula $(6 \mathrm{~mm})$ was inserted into the inferotemporal sclerotomy. After ensuring the cannula's position in the vitreous cavity, the infusion was turned on. In some cases, yellowish suprachoroidal fluid escaped from the opening during vitrectomy sclerotomies. The infusion caused the elevation of ocular pressure, resulting in more fluid escaping from the original sclerotomies [10]. For the 23-gauge/25-gauge 3-port pars plana approach, the balanced salt solution (BSS) was injected into the center of the vitreous cavity using a 29 -gauge needle to raise the intraocular pressure at the location $3.5 \mathrm{~mm}$ posterior to the limbus. A five-degree incision was made using a 23 -gauge needle to produce a scleral tunnel into the suprachoroidal space (extending no further than the suprachoroidal space) located $3.5 \mathrm{~mm}$ posterior to the limbus. When the yellowish suprachoroidal fluid was seen to escape from the site of the incision, the needle was pulled out slightly to expose the pore at the needle tip. The needle was then rotated slightly to ensure the suprachoroidal fluid to drain continuously through the scleral tunnel. If the detachment was moderate or severe, the BSS was then administered intravitreally using a 29-gauge needle connecting to the perfusion until there was no more yellowish fluid coming out, then the infusion cannula was mounted at the incision of inferotemporal sclera using a one-step procedure.

After the removal of the core vitreous by vitrectomy, a dose of $0.1 \mathrm{ml}$ Triamcinolone acetonide (TA) aqueous suspension $(40 \mathrm{mg} / \mathrm{ml}$ suspension, Erba CO., Italy) was injected into the posterior pole for visualizing the residual cortical vitreous after removing the solvent through a 0.2 $\mu \mathrm{m}$ micropore filter (Millex-GS $0.22 \mu \mathrm{g}$, Millipore). If a posterior vitreous detachment was not present, the posterior hyaloid face was then separated from the optic disk and removed using a vitreous cutter (controlled suction up to $150-500 \mathrm{mmHg}$ ). If the thin premacular vitreous/membrane was present and adhered to the retina, it was then removed using a pair of surgical forceps or a silicone-tipped needle. Perfluorocarbon liquid was injected into the eye to stabilize the retina, and the peripheral vitreous was trimmed down in all quadrants by scleral indentation. After identifying the 
retinal breaks, the subretinal fluid was removed using a soft silicone-tipped Flynn needle during fluid-gas exchange. Argon laser endophotocoagulation was performed to seal the retinal breaks. Encircling buckling was only used to repair the inferior retinal tear. Silicone oil $(\mathrm{SiO})$ tamponade was performed at the end of the surgery.

Using a permuted block design, the cases were randomly assigned with equal probability to either the oral prednisolone group or the periocular methylprednisolone injection group by a table of random digits. Each group was then divided into two subgroups according to the response of $\mathrm{CD}$ to the treatment of steroids. In the oral prednisolone group $(n=36)$, the patients took oral prednisolone $(1 \mathrm{mg} / \mathrm{kg}$ body weight) for 3-7 days before vitrectomy. In the periocular injection group $(n=39)$, the patients were administered a single periocular injection of methylprednisolone $(40 \mathrm{mg}$ ) for up to 3 days before vitrectomy. According to the response of $\mathrm{CD}$ to the treatment of steroids, each group was divided into two subgroups: A (CD subsided eyes) and subgroup B (CD persistent eyes). For the eyes did not had $\mathrm{CD}$ subsided before vitrectomy (oral prednisolone $\mathrm{B}$ group, $n=15$, and the periocular injection B group, $n=25$ ), the suprachoroidal fluid drainage was performed during vitrectomy. Postoperatively, in the oral prednisolone group, systemic steroids were tapered during a 6 -week period. Eye drops (1\% prednisolone and 1\% tropicamide) were given to all patients for 6 weeks. SiO removal was performed at 3 months after the surgery for the eyes with retinal attachment. Follow-up examinations were performed at 1 week, 1 month, 3 months, 6 months and 9 months after the vitrectomy.

Statistical analysis was performed using SPSS statistical software (version 11.5; SPSS, Chicago, IL). Continuous variables are expressed as mean \pm SD. Categorical variables are expressed as individual counts and proportions. The Snellen BCVA was converted into logarithm of minimum angle of resolution $(\log M A R)$ units for analysis. Univariate analysis was used to determine the association between anatomic and functional success after different treatments, using $\chi^{2}$ test and the Fisher's exact test, as appropriate. The critical value of significance was set at $p<0.05$ for all tests.

\section{Results}

We recruited 83 patients (83 eyes) for the study. After randomization, 41 patients were randomly assigned to the oral prednisolone group, and 42 patients were assigned to the periocular injection group. At the end of the 9-month follow-up, 75 enrolled patients ( 36 and 39 patients in the oral prednisolone group and the periocular injection group, respectively) completed the study.
Table 1 Preoperative Clinical Characteristics in Different Groups

\begin{tabular}{|c|c|c|c|}
\hline Groups & $\begin{array}{l}\text { Oral } \\
\text { prednisolone } \\
\text { group }(n=36 \\
\text { eyes) }\end{array}$ & $\begin{array}{l}\text { Periocular } \\
\text { injection group } \\
(n=39 \text { eyes })\end{array}$ & $P$ Value \\
\hline Age (years) & & & 0.272 \\
\hline Range & $38-71$ & $41-73$ & \\
\hline Mean \pm SD & $50.7 \pm 7.4$ & $52.6 \pm 7.6$ & \\
\hline Gender & & & 0.292 \\
\hline Male & $15(41.7 \%)$ & $21(53.8 \%)$ & \\
\hline Female & $21(58.3 \%)$ & $18(46.2 \%)$ & \\
\hline Visual acuity (LogMAR & units) & & 0.633 \\
\hline Range & $1.0-2.6$ & $1.0-2.6$ & \\
\hline Mean \pm SD & $1.8 \pm 0.5$ & $1.8 \pm 0.5$ & \\
\hline Duration of symptoms & & & 0.850 \\
\hline$<14$ days & $22(61.1 \%)$ & $23(58.9 \%)$ & \\
\hline 14-30 days & $14(38.9 \%)$ & $16(41.1 \%)$ & \\
\hline Lens status (eyes) & & & 0.730 \\
\hline Pseudophakic & $5(13.9 \%)$ & $4(10.3 \%)$ & \\
\hline Phakic & $31(86.1 \%)$ & $35(89.7 \%)$ & \\
\hline Extent of the RD (eyes) & & & 0.491 \\
\hline 3 quadrants & $12(33.3 \%)$ & $16(41.1 \%)$ & \\
\hline 4 quadrants & $24(66.7 \%)$ & $23(58.9 \%)$ & \\
\hline Retinal breaks number & & & 0.202 \\
\hline$\leq 2$ & $25(69.4 \%)$ & $32(82.1 \%)$ & \\
\hline$>2$ & $11(30.6 \%)$ & $7(17.9 \%)$ & \\
\hline Location & & & 0.284 \\
\hline EQ/AEQ & $28(77.8 \%)$ & $26(66.7 \%)$ & \\
\hline PEQ & $8(22.2 \%)$ & $13(33.3 \%)$ & \\
\hline Severity of CD & & & 0.575 \\
\hline Mild & $12(33.3 \%)$ & $14(35.9 \%)$ & \\
\hline Moderate & $15(41.7 \%)$ & $19(48.7 \%)$ & \\
\hline Severe & $9(25.0 \%)$ & $6(15.4 \%)$ & \\
\hline \multicolumn{4}{|l|}{ Mean IOP (mmHg) } \\
\hline Mean \pm SD & $5.3 \pm 1.2$ & $5.4 \pm 0.9$ & 0.624 \\
\hline
\end{tabular}

Oral prednisolone group: Oral prednisolone for 3-7 days; Periocular injection group: single $40 \mathrm{mg}$ periocular injection of methylprednisolone $C D$ Choroidal detachment, $R D$ retinal detachment, $E Q$ equator, $A E Q$ anterior to equator, $P E Q$ posterior to equator, $I O P$ intraocular pressure

The baseline characteristics of the oral prednisolone group and the periocular injection group were similar with respect to the preoperative variables including age, gender, visual acuity, lens status, the duration of symptoms, the extent of retinal detachment, retinal breaks (number and location), the mean intraocular pressure and the extent of PVR. The extent and severity of CD were significantly higher in the oral prednisolone group compared to that of the periocular injection group (Table 1). 
Table 2 Postoperative clinical characteristics at 9-month follow-up

\begin{tabular}{|c|c|c|c|c|c|}
\hline \multirow[t]{2}{*}{ Groups } & \multicolumn{2}{|c|}{ oral prednisolone group (36 eyes) } & \multicolumn{2}{|c|}{ periocular injection group (39 eyes) } & \multirow[t]{2}{*}{$p$ Value } \\
\hline & $\begin{array}{l}\text { oral prednisolone } \\
\text { A group ( } 21 \text { eyes) }\end{array}$ & $\begin{array}{l}\text { oral prednisolone } \\
\text { B group ( } 15 \text { eyes })\end{array}$ & $\begin{array}{l}\text { periocular } \\
\text { injection A } \\
\text { group } \\
\text { (14 eyes) }\end{array}$ & $\begin{array}{l}\text { periocular injection } \\
\text { B group ( } 25 \text { eyes })\end{array}$ & \\
\hline $\begin{array}{l}\text { Retinal reattachment } \\
\text { rate }\end{array}$ & $95.2 \%(20 / 21)$ & $86.7 \%(13 / 15)$ & $100 \%(14 / 14)$ & $92.0 \%(23 / 25)$ & 0.395 \\
\hline Final BCVA (eyes) & & & & & 0.774 \\
\hline Improved & $17(80.9 \%)$ & $13(86.7 \%)$ & $13(92.9 \%)$ & $21(84.0 \%)$ & \\
\hline Maintained & $3(14.3 \%)$ & $2(13.3 \%)$ & $1(7.1 \%)$ & $2(8.0 \%)$ & \\
\hline Worsened & $1(4.8 \%)$ & 0 & 0 & $2(8.0 \%)$ & \\
\hline \multicolumn{6}{|c|}{ Postoperative complications (eyes/total) } \\
\hline Glaucoma & $3 / 21(14.3 \%)$ & $4 / 15(26.7 \%)$ & $1 / 14(7.1 \%)$ & $7 / 25(28.0 \%)$ & 0.310 \\
\hline Cataract & $4 / 17(23.5 \%)$ & $4 / 11(36.4 \%)$ & $2 / 12(16.7 \%)$ & $8 / 19(42.1 \%)$ & 0.402 \\
\hline Inflammation & $1 / 21(4.8 \%)$ & $2 / 15(13.3 \%)$ & $2 / 14(14.3 \%)$ & $5 / 25(20.0 \%)$ & 0.462 \\
\hline \multicolumn{6}{|c|}{ Further treatment (eyes/total) } \\
\hline $\mathrm{SiO}$ removal & $17 / 21(80.9 \%)$ & $9 / 15(60.0 \%)$ & $12 / 14(85.7 \%)$ & $17 / 27(62.7 \%)$ & \\
\hline $\mathrm{SiO}$ removal $+\mathrm{Pha}$ & $3 / 21(14.3 \%)$ & $4 / 15(26.7 \%)$ & $2 / 14(14.3 \%)$ & $6 / 27(22.2 \%)$ & \\
\hline $\begin{array}{l}\mathrm{SiO} \text { removal + re- } \\
\text { vitrectomy }\end{array}$ & $1 / 21(4.8 \%)$ & $2 / 15(13.3 \%)$ & 0 & $2 / 27(7.4 \%)$ & \\
\hline $\begin{array}{l}\text { Pha after } \mathrm{SiO} \\
\text { removal }\end{array}$ & $1 / 21(4.8 \%)$ & 0 & 0 & $2 / 27(7.4 \%)$ & \\
\hline
\end{tabular}

Oral prednisolone group: patients took oral prednisolone for 3-7 days before surgery; Periocular injection group: patients were administer a single periocular injection $(40 \mathrm{mg})$ of methylprednisolone before surgery; Subgroup A: CD was subsided before surgery; Subgroup B: CD did not subsided before surgery and the eyes underwent suprachoroidal fluid drainage during surgery

$C D$ choroidal detachment, SiO Silicone oil, Pha phacoemulsification
$\mathrm{SiO}$ removal was usually performed at 3 months after surgery for the eyes with retinal attachment. If retinal detachment occurred in these eyes again, we removed the $\mathrm{SiO}$ and perform vitrectomy immediately. At 6 months after $\mathrm{SiO}$ removal, the retinal reattachment rate was $91.7 \%$ (33/36) and $94.9 \%$ (37/39) in the oral prednisolone group and the periocular injection group, respectively. There was no significant difference between the two groups $(p=0.666)$.

The retinal reattachment rate was $97.1 \%$ (34/35) and $90 \%$ (36/40), respectively, in the CD subsided eyes (the oral prednisolone A group: $n=21$; the periocular injection group $\mathrm{A}: n=15$ ) and the $\mathrm{CD}$ persistent eyes (oral prednisolone B group: $n=15$; the periocular periocular injection B group: $n=25$ ). There was no significant difference between the $\mathrm{CD}$ subsided eyes and the $\mathrm{CD}$ persistent eyes $(p=0.364)$.

The retinal reattachment rate was $95.2 \%(20 / 21), 86.7 \%$ $(13 / 15), 100 \%(14 / 14)$ and $92.0 \%(23 / 25)$ in the oral prednisolone A group, the oral prednisolone B group, the periocular injection A group, and the periocular injection B group, respectively. There was no significant difference among these groups $(p=0.395)$.
Three eyes in the oral prednisolone group ( 1 eye in the subgroup A and 2 eyes in the subgroup B) and 2 eyes in the periocular injection group B needed immediate reoperation after the removal of $\mathrm{SiO}$. Surgical procedures included membrane peeling, relaxing retinotomies, and the use of $\mathrm{SiO}$ tamponade with or without encircling buckling.

BCVA was improved in 30 eyes $(83.3 \%, 30 / 36)$ in the oral prednisolone group and 34 eyes $(87.2 \%, 34 / 39)$ in the periocular injection group, respectively. There was no significant difference between the two groups $(p=0.702)$. There was also no significant difference among the subgroups $(p=0.774)$ (Table 2). BCVA was maintained in 5 eyes $(13.9 \%)$ in the oral prednisolone group and 3 eyes (7.7\%) in the periocular injection group, respectively. Among them, one eye developed macular atrophy and two eyes developed macular pucker during the follow-up. For the 5 eyes with clear lens before surgery, 3 eyes developed nuclear sclerosis, 2 eyes developed a posterior subcapsular cataract during the follow-up period. BCVA was worsened in one eye $(2.8 \%)$ in the oral prednisolone group and 2 eyes $(5.1 \%)$ in the periocular injection group, respectively. These 3 eyes underwent prolonged period of macula detachment; 
among them, two eyes developed macular pucker and one eye developed a macular hole during the follow-up period.

There were 28 eyes and 31 eyes, in the oral prednisolone group and the periocular injection group, respectively, that were phakic at the completion of vitrectomy. Four eyes $(23.5 \%, 4 / 17)$ in the oral prednisolone A group, 4 eyes $(36.4 \%, 4 / 11)$ in the oral prednisolone B group, 2 eyes $(16.7 \%, 2 / 12)$ in the periocular injection $\mathrm{A}$ group, and 8 eyes $(42.1 \%, 8 / 19)$ in the periocular injection $\mathrm{B}$ group developed visually detrimental cataracts after surgery (Table 2). The cataract surgery was performed in these patients. The intraocular pressure was increased in 3 eyes $(14.3 \%, 3 / 21)$ in the oral prednisolone A group, 4 eyes $(26.7 \%, 4 / 15)$ in the oral prednisolone B group, one eye $(7.1 \%, 1 / 14)$ in the periocular injection $\mathrm{A}$ group, and 7 eyes $(28.0 \%, 7 / 25)$ in the periocular injection B group, respectively (Table 2). The patients were given 2 or more anti-glaucoma medications during the postoperative period. During the first week after the surgery, the rate of inflammation was 4.8, 13.3, 14.3 and $20.0 \%$, respectively, in the oral prednisolone A group, the oral prednisolone B group, the periocular injection A group, and the periocular injection B group (Table 2).

\section{Discussion}

After the onset of RRD associated with $\mathrm{CD}$, the disease develops rapidly and usually involves severe uveitis and hypotony $[9,11,12]$. When the liquefied vitreous passes through a retinal tear and is absorbed by the RPE pump, it may lead to hypotony, or stimulate the uvea to produce inflammatory mediators that cause the breakdown of the blood-retinal barrier, the exudation of excessive serum, arteriolar dilation, choroidal swelling and detachment [9, $11,12]$. These factors can cause migration of the RPE into the vitreous cavity, resulting in an inflammatory reaction that leads to PVR.

Corticosteroids may have an inhibitory effect on PVR through a variety of mechanisms, including changing the permeability of choroidal blood vessels and thereby decreasing the transudation of protein-rich fluid from inflamed choroidal vasculature [13], reducing the intraocular inflammation, stabilizing the blood-ocular barrier, suppressing the synthesis of local growth factor, and inhibiting the cellular proliferation directly $[14,15]$. Different periods of systemic steroid use have been recommended, before and after surgery, in an effort to reduce the intraocular inflammation, and more importantly, to promote the resolution of the $\mathrm{CD}[3,4,16]$. In our study, the rate of inflammation and increased intraocular pressure trended higher in the $\mathrm{CD}$ persistent eyes compared to the CD subsided eyes during the first postoperative week. In the oral prednisolone group, tapering of systemic steroids is still a routine for patients, while in the periocular injection group, the periocular injection of methylprednisolone $(40 \mathrm{mg})$ was used only once before operation. Although the steroids may reduce the inflammatory response and the incidence of PVR, the reattachment of the choroid and retina may be the most important contributor to the reduction of postoperative intraocular inflammation.

PVR is the most common cause of surgical failure in treating RRD associated with CD [17-20]. Many vitreous metabolites that contribute critically to PVR have been identified to be associated with cell proliferation. By performing primary vitreous surgery, the diseased vitreous and its mitogenic and chemotactic stimuli can be removed, leading to a high postoperative retinal reattachment rate. In addition, the suprachoroidal fluid can be drained through the pars plana sclerotomy, and the retinal breaks can be easily identified during the surgery since the $\mathrm{CD}$ may be hidden in the small retinal folds.

A recent clinical multicenter study showed that $C D$ was an independent explanatory variable for surgical failure of the retinal detachment repair [18]. Without prompt treatment, the prognosis of $\mathrm{CD}$ was poor because of the rapid onset of massive vitreous and periretinal proliferation [4]. Although the prolonged macular detachment caused by a few days of postponement of surgery may not lead to poor visual outcomes, it is not necessary to use systemic steroids before and after operation, and delay the surgery.

In summary, for RRD combined with $\mathrm{CD}$, a single periocular injection of methylprednisolone ( $40 \mathrm{mg}$ ) 1-3 days before PPV combined with the drainage of suprachoroidal fluids during the surgery results in similar anatomic and functional outcomes compared to the treatment of oral prednisolone for 3-7 days before vitrectomy. Our findings suggest that it may not be necessary to use systemic steroids and wait for $\mathrm{CD}$ to be completely subsided before the vitrectomy in the patients with RRD combined with $\mathrm{CD}$.

\section{Summary}

\section{What was known before}

- The systemic (oral prednisolone) / or local (periocular/ intravitreal) corticosteroids for 3-7 days before PPV was an acceptable alternative for treatment of rhegmatogenous retinal detachment combined with choroidal detachment.

\section{What this study adds}

- If single periocular injection of methylprednisolone and drainage of suprachoroidal fluids during vitrectomy has a similar retinal reattachment rate, it would not be 
necessary to use systemic steroids and delay surgery on the treatment of rhegmatogenous retinal detachment combined with choroidal detachment.

Funding This study was supported in part by The Eye Hospital of Wenzhou Medical University.

\section{Compliance with ethical standards}

Conflict of interest The authors declare that they have no conflict of interest.

Publisher's note: Springer Nature remains neutral with regard to jurisdictional claims in published maps and institutional affiliations.

\section{References}

1. Zhu J, Xu X, Zhang X. Surgical therapeutic results of rhegmatogenous retinal detachment associated with choroidal detachment. Zhonghua Yan Ke Za Zhi. 2002;38:135-9.

2. Li Z, Li Y, Huang X, Cai XY, Chen X, Li S, et al. Quantitative analysis of rhegmatogenous retinal detachment associated with choroidal detachment in Chinese using UBM. Retina. 2012;32:2020-5.

3. Gottlieb F. Combined choroidal and retinal detachment. Arch Ophthalmol. 1972;88:481-6.

4. Seelenfreund MH, Kraushar MF, Schepens CL, Freilich DB. Choroidal detachment associated with primary retinal detachment. Arch Ophthalmol. 1974;91:254-8.

5. Sharma T, Challa JK, Ravishankar KV, Murugesan R. Scleral buckling for retinal detachment predictors for anatomic failure. Retina. 1994;14:338-43.

6. Yang CM. Pars plana vitrectomy in the treatment of combined rhegmatogenous retinal detachment and choroidal detachment in aphakic or pseudophakic patients. Ophthalmic Surg Lasers. 1997;28:288-93.

7. Sharma T, Gopal L, Badrinath SS. Primary vitrectomy for rhegmatogenous retinal detachment associated with choroidal detachment. Ophthalmology. 1998;105:2282-5.

8. Ghoraba HH. Primary vitrectomy for the management of rhegmatogenous retinal detachment associated with choroidal detachment. Graefes Arch Clin Exp Ophthalmol. 2001;239: 733-6.

9. Sharma T, Gopal L, Reddy RK, Kasinathan N, Shah NA, Sulochana KN, et al. Primary vitrectomy for combined rhegmatogenous retinal detachment and choroidal detachment with or without oral corticosteroids: a pilot study. Retina. 2005;25:152-7.

10. Wei Y, Wang N, Chen F, Wang H, Bi C, Zu Z, et al. Vitrectomy combined with periocular/intravitreal injection of steroids for rhegmatogenous retinal detachment associated with choroidal detachment. Retina. 2014;34:136-41.

11. Kang JH, Park KA, Shin WJ, Kang SW. Macular hole as a risk factor of choroidal detachment in rhegmatogenous retinal detachment. Korean J Ophthalmol. 2008;22:100-3.

12. Jarrett WH II. Rhematogenous retinal detachment complicated by severe intraocular inflammation, hypotony, and choroidal detachment. Trans Am Ophthalmol Soc. 1981;79:664-83.

13. Cook C, Macdonald RK. Effect of cortisone on the permeability of the blood-aqueous barrier to fluorescein. Br J Ophthalmol. 1951;35:730-40.

14. Rubsamen PE, Cousins SW. Therapeutic effect of periocular corticosteroids in experimental proliferative vitreoretinopathy. Retina. 1997; 17:44-50.

15. Ando N, Sen HA, Berkowitz BA, Wilson CA, de Juan E Jr. Localization and quantitation of blood-retinal barrier breakdown in experimental proliferative vitreoretinopathy. Arch Ophthalmol. 1994;112:117-22.

16. Brockhurst RJ, Schepens CL, Okamura ID, Regan CD, McMeel JW. Scleral buckling procedures. 8. Preoperative complications. Arch Ophthalmol. 1965;74:792-8.

17. Yu M, Wu Z, Zhang Z, Huang X, Zhang Q. Metabolomic analysis of human vitreous in rhegmatogenous retinal detachment associated with choroidal detachment. Invest Ophthalmol Vis Sci. 2015;56:5706-13.

18. Dai Ying, Wu Zhifeng, Sheng Huiming, Zhang Zhengwei, Yu Mengxi, Zhang Qing. Identification of inflammatory mediators in patients with rhegmatogenous retinal detachment associated with choroidal detachment. Mol Vis. 2015;21:417-27.

19. Ricker LJ, Kessels AG, de Jager W, Hendrikse F, Kijlstra A, la Heij EC. Prediction of proliferative vitreoretinopathy after retinal detachment surgery: potential of biomarker profiling. Am J Ophthalmol. 2012;154:347-54.

20. Adelman RA, Parnes AJ, Michalewska Z, Ducournau D, European Vitreo-Retinal Society Retinal Detachment Study Group. Clinical variables associated with failure of retinal detachment repair: the European Vitreo-Retinal Society retinal detachment study report number 4. Ophthalmology. 2014;121:1715-9. 